\title{
LAVADO PULMONAR TOTAL BILATERAL EN UNA SOLA SESIÓN BAJO ECMO VENO-VENOSO EN UN CASO PROTEINOSIS ALVEOLAR SEVERA: CINCO AÑOS DE SEGUIMIENTO.
}

\section{BILATERAL SINGLE SESION WHOLE LUNG LAVAGE UNDER VENOUS-VENOUS ECMO IN A CASE OF SEVERE PULMONARY ALVEOLAR PROTEINOSIS: FIVE YEARS FOLLOW UP.}

\author{
Esteban Javier Wainstein ${ }^{1,4}$, Marcos José Las Heras ${ }^{1,2}$, Jorge Federico Sinner ${ }^{2}$, Vadim Kotowicz ${ }^{3}$, \\ Juan Arturo Precerutti ${ }^{1}$, Juan Eduardo San Román ${ }^{2}$, Graciela Noemí Svetliza ${ }^{1}$
}

\begin{abstract}
1. Sección Neumonología, Servicio de Clínica Médica, Hospital Italiano de Buenos Aires

2. Servicio de Terapia Intensiva de Adultos, Hospital Italiano de Buenos Aires

3. Servicio de Cirugía Cardiovascular, Hospital Italiano de Buenos Aires.

4. Email de contacto: esteban.wainstein@hospitalitaliano.org.ar
\end{abstract}

\section{Conceptos claves:}

A) Qué se sabe sobre el tema: la proteinosis alveolar es una enfermedad infrecuente causada por la acumulación de surfactante en los espacios alveolares debido a diversos mecanismos. El lavado pulmonar total es el tratamiento standard. Pocos casos han sido descriptos que requieran soporte vital extracorpóreo para tal tratamiento.

B) Qué aporta este trabajo: describimos un caso de proteinosis alveolar severa en el que se realizó un lavado pulmonar total exitoso en una sola sesión bajo ECMO veno-venoso y que se mantiene asintomático tras 5 años de seguimiento. Mostramos la factibilidad del uso de ECMO venoso en lugar del ECMO veno arterial.

Recibido: 2018-12-08 Aceptado: 2018-12-16 DOI: http://dx.doi.org/10.31053/1853.0605.v76.n2.22595 (c) (i) (8)

(c) Universidad Nacional de Córdoba

\section{Resumen:}

Introducción: la proteinosis alveolar es una enfermedad rara caracterizada por la acumulación de material proteináceo en los espacios alveolares. El principal mecanismo implicado es el desarrollo de anticuerpos contra el factor estimulante de colonias de granulocitos y macrófagos (GM-CSF), que impide la activación del macrófago alveolar y genera acumulación de surfactante. Su tratamiento estándar es el lavado pulmonar total (LPT).

Objetivo: presentar el caso de una mujer de 47 años derivada para trasplante pulmonar por sospecha de combinación de fibrosis y enfisema. Se realizó el diagnóstico insuficiencia respiratoria por proteinosis alveolar pulmonar severa y se realizó tratamiento con lavado pulmonar total bilateral, secuencial y en una sola sesión bajo ECMO veno-venoso. A 5 años del tratamiento, la paciente se encuentra asintomática y con fisiología pulmonar normal.

Conclusiones: el caso resalta la importancia de evaluar cuidadosamente el diagnóstico de todos los pacientes, en especial los que son derivados a trasplante pulmonar y muestra la factibilidad de realizar el lavado pulmonar total bajo ECMO veno-venoso sin producir claudicación del ventrículo derecho.

Palabras clave: Oxigenación por membrana extracorporea ; enfermedades pulmonares intersticiales ; insuficiencia respiratoria

\section{Abstract:}

Introduction: Pulmonary alveolar proteinosis (PAP) is a rare disease caused by accumulation of proteinaceous material in the alveolar spaces. The most common mechanism implied is the development of antibodies directed to granulocyte macrophage colony stimulating factor (GM-CSF). which inhibits activation of alveolar macrophage, thereby causing accumulation of surfactant. Standard treatment is considered to be whole lung lavage (WLL).

Object: We present the case of a 47 years old woman referred for lung transplantation because of combined lung fibrosis and emphysema. Diagnose was change to respiratory failure secondary to PAP. We performed a single cession bilateral WLL under venousvenous ECMO. After a 5 years follow up, the patients remains asymptomatic and with normal pulmonary physiology.

Conclusions: The case highlights the importance of careful examination of previous diagnoses, in particular of cases referred for lung transplantation and provides factibility of performing single session bilateral WLL without causing right heart failure.

Keywords: extracorporeal Membrane Oxygenatio, lung Diseases, Interstitial, Respiratory Insufficiency 


\section{Introducción}

La proteinosis alveolar es una enfermedad rara, caracterizada por la acumulación de material proteináceo en los espacios alveolares. El principal mecanismo implicado es el desarrollo de anticuerpos contra el factor estimulante de colonias de granulocitos y macrófagos (GM-CSF), que impide la activación del macrófago alveolar y genera acumulación de surfactante. En forma más infrecuente, es secundaria a neoplasias hematológicas, algunas infecciones o exposición al sílice ${ }^{1,2}$. Su tratamiento estándar es el lavado pulmonar total (LPT) ${ }^{2}$. Raramente es diagnosticada en etapas tan avanzadas que requieran soporte vital extracorpóreo para sostener el LPT ${ }^{3}$. El LPT bajo circulación extracorpórea y más recientemente con oxigenación bajo membrana extracorpórea $(\mathrm{ECMO})$ ha sido descripto, pero excepcionalmente realizado en modalidad de ECMO veno-venoso por potencial claudicación del ventrículo derecho ${ }^{3,4,5}$. La evolución posterior de esos casos graves es escasamente conocida ${ }^{2}$.

\section{Caso Clínico}

Una mujer de 40 años, fumadora, fue derivada a nuestro centro para ser evaluada como potencial candidata a recibir un trasplante pulmonar por sospecha de síndrome combinado de enfisema-fibrosis. La disnea llevaba 4 años de evolución y había sido progresiva hasta clase funcional IV. Al ingreso se encontraba lúcida, en buen estado general, con dedos en palillo de tambor y una saturación de oxígeno por saturometría de pulso de $87 \%$ respirando a través de una máscara con reservorio. La tomografía computada de tórax evidenció imágenes típicas de proteinosis alveolar: patrón en crazy paving difuso y bilateral, con menor afectación del manto y consolidaciones en ambos lóbulos inferiores (figura 1). Se realizó un lavado broncoalveolar que confirmó el diagnóstico, con el aspecto macroscópico típico y la citología característica. Los anticuerpos anti GM-CSF fueron negativos. Se decidió proceder a conexión a asistencia respiratoria para proceder a un LPT. La presión arterial de oxígeno era de 62 $\mathrm{mmHg}$ bajo una fracción inspirada de oxígeno del 100\%. Dado la severidad del cuadro, realizamos un LPT bajo soporte con ECMO veno-venoso en forma bilateral y en una sola sesión. Se realizó una canulación periférica yugular con cánula número 22, una canulación femoral con una cánula número 25 y se instauró un flujo de 4 litros por minuto. Se infundieron 18 litros de solución salina al pulmón derecho y 17 litros al pulmón izquierdo (figura 2). El procedimiento se realizó sin inestabilidad hemodinámica, bajo monitoreo hemodinámico con vías arterial y central. La paciente logró desvincularse del ECMO a las 36 hs y de la asistencia respiratoria mecánica a los 5 días. No presentó complicaciones. La clínica, la radiología y los estudios funcionales mostraron mejoría progresiva durante los meses siguientes. A 5 años del tratamiento, se encuentra en clase funcional I, con mínima alteración radiológica (figura 3) y una capacidad de difusión (DLco) del 80\% del valor predicho.
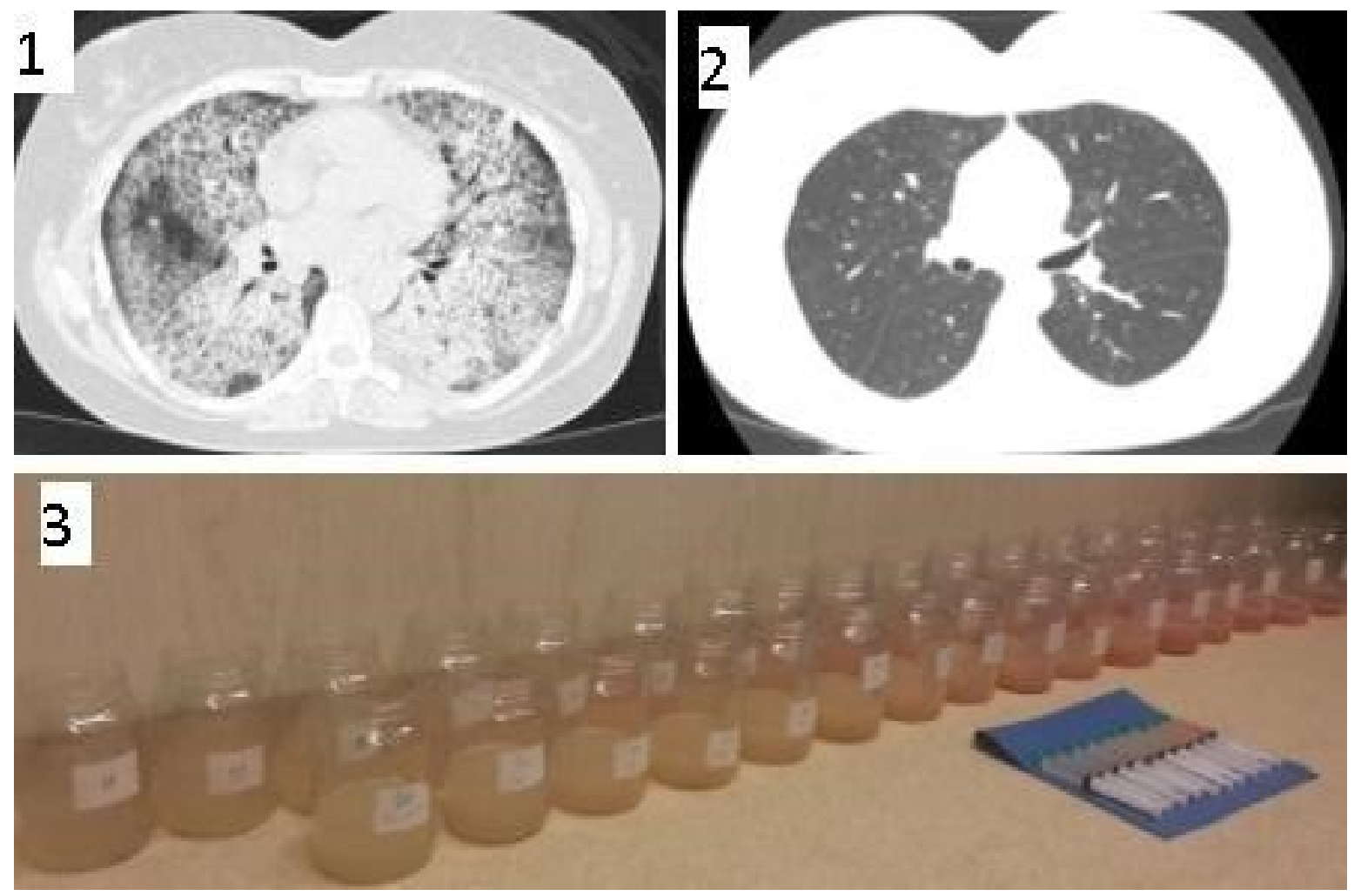

Figura 1: tomografía previa al LPT. Figura 2: fotografía del material del LPT. Figura 3: tomografía a 5 años del LPT 


\section{Discusión}

Formas severas de proteinosis alveolar, aunque bien descriptas, son infrecuentes ${ }^{3}$. EI LPT ha sido bien descripto bajo soporte vital con circulación extracorpórea y más recientemente $\mathrm{ECMO}^{2,5,6}$. La decisión de realizar LPT bajo ECMO veno-venoso en lugar de venoarterial puede ser controversial por el potencial fallo del ventrículo derecho, pero en este caso fue bien tolerado. La modalidad venovenosa es de aplicación más sencilla y con menores complicaciones que la modalidad veno-arterial ${ }^{4}$. El motivo de la mejoría progresiva posterior no es desconocido. Si bien podría atribuirse al abandono del tabaquismo, también ha sido previamente hipotetizado que los lavados pulmonares totales bajo ECMO son más agresivos, permitiendo mayores intervalos libres de recaída3. Al no presentar anticuerpos anti GMS-CSF deberíamos considerar causas secundarias de proteinosis alveolar ${ }^{1}$. Tras 5 años de seguimiento, parece improbable que sea secundaria a enfermedades hematológicas. La paciente se desempeñaba trabajando en un lavadero de ropa: la exposición a detergentes que potencialmente podrían alterar el recambio de surfactante como causa de proteinosis alveolar nos resulta interesante pero meramente especulativa.

\section{Conclusión}

El caso presentado resalta la necesidad de evaluar el diagnóstico de todos los pacientes, en especial aquellos que son derivados como potenciales candidatos a recibir un trasplante pulmonar. Además, deja otro antecedente acerca de la factibilidad y el éxito a largo plazo de realizar el lavado pulmonar total en una sola sesión bajo ECMO veno-venoso en casos de proteinosis alveolar severa.

Limitaciones de responsabilidad

El presente trabajo representa a los autores y no a la institución donde trabajan. No tuvo fuentes de financiación.

Conflictos de interés

No existen conflictos de interés.

\section{Bibliografía}

1. Bruce C. Trapnell, Jeffrey A. Whitsett, Koh Nakata. Pulmonary Alveolar Proteinosis. N Engl J Med 2003; 349:2527-2539.

2. José Antonio Rodríguez Portal. Tratamiento de la proteinosis alveolar primaria del adulto. Arch Bronconeumol 2015;51:344-9.

3. John F. Seymour, Jeffrey J. Presneil. Pulmonary Alveolar Proteinosis. Progress in the First 44 Years. Am J Respir Crit Care Med 2002;166:215-35.

4. Elliott S. Cohen, Ellen Elpern, Michael R. Silver. Pulmonary Alveolar Proteinosis Causing Severe Hypoxemic Respiratory Failure Treated With Sequential Whole-Lung Lavage Utilizing Venovenous Extracorporeal Membrane Oxygenation: A Case Report and Review. Chest. 2001;120:1024-26.

5. Tomas Vymazal, Martina Krecmerova. Respiratory Strategies and Airway Management in Patients with Pulmonary Alveolar Proteinosis: A Review. Biomed Res Int. 2015; 2015: 639543.

6. Krecmerova M, Mosna F, Bicek V. Petrik F, Grandcourtova A, Lekes $M$, et al. Extracorporeal membrane oxygenation to support repeated whole-lung lavage in a patient with pulmonary alveolar proteinosis in life threatening dyspnoe-a case report. BMC Anesthesiol. 2015; 30;15:173. 